\title{
A microRNA delivery carrier for hepatic carcinoma therapy using layer-by-layer self-assembled mesenchymal stem cells
}

\author{
Chunmin $\mathrm{Li}^{1 \#}$, Xin $\mathrm{Li}^{2 \#}$, Fengsheng $\mathrm{Li}^{3}$, Dawei Wang ${ }^{1}$, Jin Wang ${ }^{3}$, Tao Yan ${ }^{3}$, Bo Sun ${ }^{3}$, Hualiang Ren ${ }^{1}$ \\ ${ }^{1}$ Department of Vascular Surgery, Beijing Chao-Yang Hospital, Capital Medical University, Beijing, China; ${ }^{2}$ Handan First Hospital, Handan, \\ Handan, China; ${ }^{3}$ PLA Rocket Force Characteristic Medical Center, Beijing, China \\ Contributions: (I) Conception and design: C Li, H Ren; (II) Administrative support: B Sun; (III) Provision of study materials or patients: B Sun; (IV) \\ Collection and assembly of data: X Li, D Wang, J Wang, T Yan; (V) Data analysis and interpretation: C Li, F Li, H Ren; (VI) Manuscript writing: All \\ authors; (VII) Final approval of manuscript: All authors. \\ \#These authors contributed equally to this work. \\ Correspondence to: Hualiang Ren. Department of Vascular Surgery, Beijing Chao-Yang Hospital, Capital Medical University, 5 Gongren Tiyuchang \\ Nanlu, Beijing, 100020, China. Email: renhualiang@outlook.com; Bo Sun. PLA Rocket Force Characteristic Medical Center, 16 Xin Jie Kou Wai \\ Street, Beijing 100088, China. Email: sunbo801025@163.com.
}

Background: Several microRNAs (miRNAs), such as miR101, have been reported to be effective for
hepatocellular carcinoma (HCC) therapy in preclinical studies; while its further application is hampered due
to the lack of desirable delivery systems. Based on the characteristics of mesenchymal stem cells (MSCs),
including good biocompatibility and tropism to HCC, the current study was designed to investigate whether
miR101-loaded MSCs (miR101-MSCs) using layer-by layer (LbL) self-assembled gelatin and alginate could
target the delivery of miR101 to HCC.
Methods: The characterization of miR101-loaded MSCs was determined by transmission electron
microscopy (TEM), scanning electron microscopy (SEM) and Zeta potential analysis. The tropism and
of miR101-MSCs to HCC were detected by Transwell chamber assay, and the anti-tumor potential of
miR101-MSCs against HCC was examined by Annexin V-FITC/PI staining, BrdU incorporation assay, and
immunoblotting with antibodies against proliferating cell nuclear antigen (PCNA) and caspase-3.
Results: The results showed that a thin LbL film containing miR101 was encapsulated on the surface of
MSCs. Furthermore, miR101-loaded MSCs had a tropism to hepatoma cells. Finally, treatment of BEL-
7402 cells, an HCC cell line, with miR101-loaded MSCs led to significant proliferation inhibition and
apoptosis of BEL-7402 cells.

Conclusions: These in vitro findings suggest that MSCs loaded with miRNA by LbL self-assembly may be a promising and minimally invasive approach for targeted treatment of HCC.

Keywords: MicroRNA; layer-by layer self-assembly; mesenchymal stem cells (MSCs); hepatic carcinoma; targeted delivery

Submitted Jul 24, 2019. Accepted for publication Mar 03, 2020.

doi: $10.21037 /$ tcr-19-1378b

View this article at: http://dx.doi.org/10.21037/tcr-19-1378b

\section{Introduction}

Hepatocellular carcinoma (HCC) is the second most common malignant tumor-related death in males in China according to the report of the World Health Organization on the cancer country profiles of 2014, threatening the health of humans. MicroRNA (miRNA), a type of endogenous noncoding RNA with a length of 21-30 nucleotides, can down-regulate the expression of various target genes by binding to their mRNAs. It has been established that the expression of miRNAs is aberrant in various types of tumors, such as HCC, leading to the 
activation of the oncogenic pathway or inactivation of the anti-oncogenic pathway (1). MiR101 has been established to be an tumor-suppressive miRNA, and significantly downregulated in HCC, which contributed to the decreased apoptosis, increased proliferation, invasiveness and chemoresistant of HCC (2-5). MiR101 effectively prevents liver tumor development $(2,4)$, providing strong evidence that miR-101 may be an ideal candidate for miRNA-based treatment for HCC.

Although systemic or local delivery of miRNA mimics, precursors or expression vectors has been considered a promising strategies for cancer treatment, several miRNAbased therapies, such as MiR-34a and MiR-16, have entered into phase I clinical trials for the treatment of primary liver cancer or malignant pleural mesothelioma (6). The clinical success of miRNA-based therapies has been limited due to the lack of an appropriate delivery system (7). For instance, both the cationic lipid-based system and the most widely investigated polyethylenimine (PEI)-based delivery system showed significant toxicity, nonspecific delivery, or unwanted immune response in vivo (8-10). To address this challenge, various miRNA delivery strategies have been extensively investigated. Xie and his colleagues (11) developed self-immolative nanoparticles that can effectively deliver miRNA after intratumoral injection in vivo and show no apparent signs of gross toxicity, while the limitation of nonspecific delivery remains outstanding. Tivnan et al. (12) reported that nanoparticles containing therapeutic miRNA, conjugated with an antibody against the antigen that was highly expressed on the cancer cell surface, could result in tumor-specific delivery of miRNA after systemic administration into mice. However, the issue of toxicity presented in other antibody drug conjugate research, such as hematologic, hepatic and neurologic damage (13), might also be observed when using this strategy for the targeted delivery of miRNA.

Mesenchymal stem cells (MSCs) were recently found to possess tumor-tropic capacity, in addition to multipotent differentiation capabilities (14-16). Although the mechanism underlying tumor tropism of MSCs is not fully understood, the microenvironment of HCC, which could recruit MSCs by tumor cells-secreted various soluble factors (17), such as stromal-derived factor-1 (SDF-1), platelet-derived growth factor (PDGF), epidermal growth factor (EGF), matrix metalloproteinase-1 (MMP-1), and macrophage chemoattractant protein-1 (MCP-1) $(18,19)$, has been found to be responsible for the tumor homing of
MSCs. The tumor-tropic characteristic of MSCs, as well as good biocompatibility due to autogenous resources, enables them to be used as vehicles for delivering anticancer agents, including anticancer genes $(20,21)$.

Layer-by-layer (LbL) self-assembly of films is widely used in various fields, including the delivery of biomolecules, such as insulin (22), doxorubicin (23), and bone morphogeneticprotein-2 (BMP-2) (24). By alternating the coating of oppositely charged biocompatible materials that could be loaded with biomolecules, the LbL technique can form films onto the surface of cells or bioengineered grafts. More recently, Li et al. (25) reported that, using the positively charged gelatin (type A) and negatively charged alginate, both of which are biodegradable, single neural stem cells (NSCs) can be successfully encapsulated by the LbL technique. More importantly, insulin-like growth factor-1 (IGF-1) loaded on alginate can be released into medium in a time- and $\mathrm{pH}$-dependent manner, while the viability, proliferation and differentiation of NSCs remain unaffected (25). These findings encouraged us to explore whether miR101-loaded MSCs by LbL self-assembly with polyelectrolytes are good targeting vehicles for miR101 to HCC.

The aim of this study was to determine whether miR101 can be loaded on the surface of MSCs by the LbL selfassembly technique using gelatin and alginate containing miR101 and whether these miR101-loaded MSCs can tropistically migrate to HCC and suppress the growth of HCC in vitro.

\section{Methods}

\section{Cell culture}

Rat MSCs separated from the bone marrow of SD rat and cultured at the second passage were purchased from Cyagen (Santa Clara, CA, USA). Cells cultured in $\alpha$-MEM medium (HyClone, Logan, Utah) with 10\% fetal bovine serum (FBS) and antibiotics were used in subsequent experiments.

BEL-7402 HCC cells (Num. HLCL-003) were purchased from iCell Bioscience Inc. (Shanghai, China) and cultured in F12k medium (Sigma-Aldrich, St. Louis, MO, USA) supplemented with $15 \%$ horse serum (Thermo Fisher Scientific Inc., Waltham, MA, USA), 2.5\% FBS (Thermo Fisher Scientific Inc., Waltham, MA, USA) and $100 \mathrm{IU} / \mathrm{mL}$ penicillin/streptomycin. The cells were cultured at $37{ }^{\circ} \mathrm{C}$ in an incubator containing $5 \% \mathrm{CO}_{2}$. 
Preparation of miR101-loaded MSCs (miR101-MSCs)

miR101 (5'-CCGGAATTCAATGACCTCTTTTCTT CTGC-3'), synthesized by GenePharma Co. (Shanghai, China), was dissolved in $0.1 \%$ alginate to prepare an alginate-miR101 mixed solution containing $0.05 \% \mathrm{miR}$ 101. The miR101-loaded MSCs were assembled using the layer-by-layer technique with gelatin and alginatemiR101 as previously reported $(26,27)$. Briefly, $5 \times 10^{6}$ MSCs suspended in $5 \mathrm{~mL}$ of $0.05 \%$ gelatin solution were shaken for $5 \mathrm{~min}$ at $37^{\circ} \mathrm{C}$, followed by centrifugation at 2,000 rpm for $5 \mathrm{~min}$. After washing with $10 \mathrm{~mL}$ of phosphate-buffered saline (PBS), the MSCs were incubated with $5 \mathrm{~mL}$ of alginate-miR101 mixed solution on a shaker for $5 \mathrm{~min}$ at $37^{\circ} \mathrm{C}$, followed by washing with PBS. The cells were resuspended in gelatin solution as described above to coat another layer of gelatin. Control-MSCs (Ctl-MSCs) were prepared as described above, except for the miR101 loading procedure, during which the MSCs were incubated with $0.1 \%$ alginate without miR101.

\section{Analysis of miR101-MSCs using transmission electron microscopy (TEM)}

miR101-MSCs were morphologically analyzed using TEM. Briefly, after fixation with $2.5 \%$ glutaraldehyde in $0.1 \mathrm{M}$ Sorensen's buffer and subsequent washing with $5 \%$ sucrose solution in $0.1 \mathrm{M}$ Sorensen's buffer 3 times, MSCs and miR101-MSCs were fixed with $1 \% \mathrm{OsO}_{4}$ for $2 \mathrm{~h}$, followed by a procedure of dehydration using graded alcohols and methanol. The cells were subsequently embedded in epoxy resin to prepare thin sections using a microtome (318423, Reichert Nr.). Finally, the samples mounted on copper grids were stained with $2 \%$ uranyl acetate combined with $1 \%$ lead citrate before analysis using TEM (CM-10, Philips, The Netherlands).

\section{Morphological analysis of miR101-loaded MSCs using scanning electron microscopy (SEM)}

miR101-MSCs were fixed and dehydrated using the method described for the TEM analysis. After spraying with a gold coat, the cells were viewed using SEM (JEOL 5900).

\section{Zeta potential of miR101-loaded MSCs}

The zeta potential of miR101-MSCs and Control-
MSCs was determined using a zeta potential analyzer (Brookhaven).

\section{Scratch assays}

Scratch assays were performed according to previously published methods (28). Briefly, MSCs grown in sixwell plates with $95 \%$ confluence were further cultured in $\alpha$-MEM medium without FBS for $24 \mathrm{~h}$. A scratch in the cell layer was performed using a $200 \mu \mathrm{L}$ pipet tip, followed by washing with PBS for 3 times to remove debris. The cells were grown in FBS-free $\alpha$-MEM medium for $72 \mathrm{~h}$. Images were taken under a microscope at 0 and $72 \mathrm{~h}$ after the scratch, and the distance of gap was determined by ImagePro Plus. The rate of gap closure (RGC) was calculated the following formula.

RGC $(\%)=\left(\mathrm{D}_{0}-\mathrm{D}_{72}\right) / \mathrm{D}_{0}$

Where $\mathrm{D}_{0}$ represents the distance of gap at $0 \mathrm{~h}$, while $\mathrm{D}_{72}$ represents the distance at $72 \mathrm{~h}$.

\section{Tropism detection of miR101-loaded MSCs to HCC}

The tropism of miR101-MSCs to HCC was performed in vitro using a Transwell coculture system (pore size: $8 \mu \mathrm{m}$, Corning Costar, USA). A total of $1 \times 10^{5} \mathrm{miR} 101-$ MSCs or untreated MSCs in $500 \mu \mathrm{L}$ of medium were added to the upper chambers, and the lower chambers were supplemented with $1.5 \mathrm{~mL}$ of F12k medium with or without $1.5 \times 10^{6}$ BEL-7402 cells. After incubation in a humidified incubator $\left(37^{\circ} \mathrm{C}, 5 \% \mathrm{CO}_{2}\right)$ for $96 \mathrm{~h}$, the miR101-MSCs or untreated MSCs attaching on the lower surface the membrane were stained with crystal violet. The average number of cells in five random 100x magnification fields under the microscope was counted.

\section{Apoptosis of tumor induced by miR101-loaded MSCs in vitro}

A Transwell coculture system was used to detect the apoptosis of tumors induced by miR101-loaded MSCs. Similar to the tropic detection of miR101-MSCs to HCC described above, $1 \times 10^{5} \mathrm{miR} 101-$ or Ctl-MSCs in $500 \mu \mathrm{L}$ of medium and $1.5 \times 10^{6}$ BEL-7402 cells in $1.5 \mathrm{~mL}$ of F12k medium were seeded into the upper chamber and lower chamber, respectively. Twenty-four hours after incubation, the BEL-7402 cells in the lower chamber were collected by digestion with $0.25 \%$ trypsin and subjected to a cell 
apoptosis assay using the annexin V-FITC (fluorescein isothiocyanate) Apoptosis Detection Kit (Bosai, China) according to the manufacturer's instructions. The cells were finally analyzed by a flow cytometer (BD Accuri C6, Becton Dickinson, San Jose, CA, USA).

\section{Proliferation assay using BrdU incorporation assay}

Proliferation detection was also performed using the Transwell coculture system and BrdU incorporation assay. Briefly, $5 \times 10^{3}$ BEL-7402 cells in $1.5 \mathrm{~mL}$ of F12k medium were seeded into the lower chamber, and $5 \times 10^{3} \mathrm{miR} 101-$ or Ctl-MSCs in $500 \mu \mathrm{L}$ medium were seeded into the upper chamber. The coculture system was incubated for $24 \mathrm{~h}$. Proliferation analysis was performed using BrdU-cell proliferation ELISA (Roche Diagnostics GmbH, Penzberg, Germany) according to the manufacturer's protocol. Images were taken in 10 random fields using a fluorescence microscope (TE2000-E, Nikon). Positive cells were quantified by Image-Pro Plus (V6.0 Media Cybernetics).

\section{Immunoblotting}

The BEL-7402 cells in the lower chamber were harvested $48 \mathrm{~h}$ after the Transwell coculture system was established as described above. The cells were then lysed in lysis buffer to obtain the total protein content, whose concentration was detected by the BCA method. Equal amounts $(50 \mu \mathrm{g}$ protein/lane) of proteins were subjected to sodium dodecyl sulfate-polyacrylamide gel electrophoresis (SDS-PAGE) analysis, and transferred to the nitrocellulose membranes. After blocking with 5\% non-fat milk, the membranes were incubated with primary antibodies against proliferating cell nuclear antigen (PCNA, 1:1,000 dilution, ImmunoWay Biotechnology Company, Plano, USA), caspase-3 (1:2,000 dilution, Cell Signaling Technology, Shanghai, China) or $\beta$-tubulin (1:5,000 dilution, YTHX Biotechnology Co. Ltd., Beijing, China), followed by 1-hour incubation with peroxidase (HRP)-conjugated secondary antibody at room temperature. An enhanced chemiluminescence kit (ECL, Thermo Fisher Scientific, Waltham, USA) was used to visualize the bands.

\section{Statement of ethics approval}

The ethics approval is not required because no primary data collected from humans or animal.

\section{Statistical analysis}

The experimental data were analyzed by SPSS 18.0. Variance analyses were performed, and the results are expressed as the means and error of the means (EM). Each experiment was repeated at least three times. All $\mathrm{P}$ values were two-tailed, and a $\mathrm{P}<0.05$ was considered significant.

\section{Results}

\section{Characterization of miR101-loaded MSCs}

To investigate whether the LbL film was coated on the MSCs, miR101-MSCs were subjected to TEM. As shown in Figure $1 A$, a LbL thin film with a thickness of approximately $5.8 \pm 2.2 \mathrm{~nm}$, which was calculated by ImageJ, was observed on the surface of miR101-MSCs rather than the untreated MSCs, which indicated that MSCs were successfully encapsulated by gelatin and alginate-miR101.

The miR101-MSCs were analyzed using SEM on days 1, 3 and 7 . The results showed that the attachment of miR101loaded MSCs was slower than that of untreated MSCs, and miR101-MSCs showed a spheroid-like morphology on days 1 and 5, while MSCs presented a spindle-like morphology. On day 7, the miR101-MSCs took on a spindle-like morphology, which was similar to that of MSCs on day 1 (Figure 1B).

Previous study had shown that the zeta potential of cells changed with different layers of gelatin and alginate (29). We therefore detected the zeta potential of miR101MSCs during LbL self-assembly of films. As shown in Figure $1 C$, the zeta potential of the MSCs changed from -40 to $5 \mathrm{mV}$ after coating with positively charged gelatin, and subsequently returned to $-40 \mathrm{mV}$ when the cells were further coated with negatively charged alginate. Finally, the zeta potential of the MSCs changed to $5 \mathrm{mV}$ after the third layer of gelatin film was coated. These results indicate that the oppositely charged polyelectrolytes can complementary bound together and coat on the surface of MSCs in a manner of LbL.

\section{Tumor-tropism of miR101-MSCs to HCC was slightly weakened in vitro}

The effect of LbL film on the migration of MSCs was first investigated by scratch assay. As shown in Figure $2 A, B$, the results showed no significant difference in the migration of untreated MSCs and miR101-MSCs. 
A

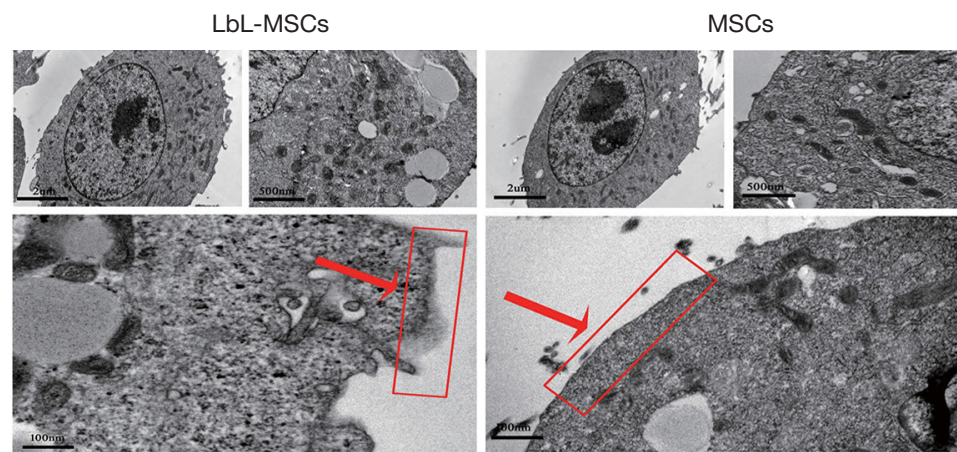

B
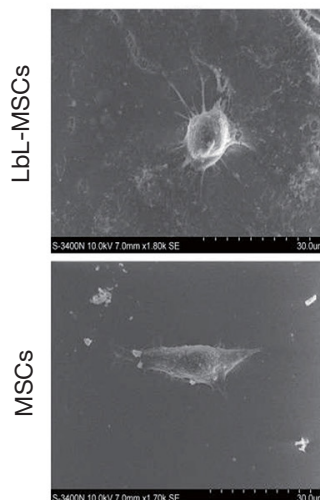

$3 d$

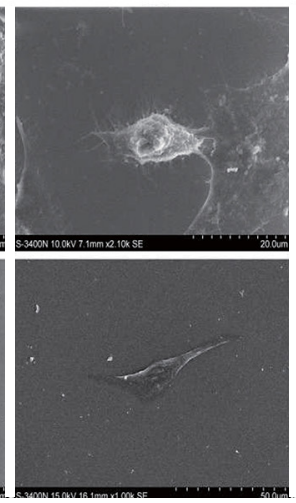

$7 d$

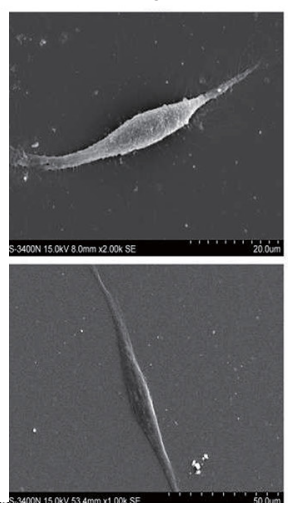

C

Figure 1 Physical characterization of miR101-MSCs. miR101-MSCs and untreated MSCs were subjected to morphological analysis using TEM and SEM or zeta potential detection. (A) TEM images of miR101- (left panel) and untreated (right panel) MSCs with different magnifications. Arrows indicate the MSC surface with or without the thin LbL film. (B) SEM images of miR101-MSCs (upper panel) and untreated MSCs (lower panel) on days 1,3 and 7. (C) Zeta potential of MSCs coated with different layers of materials.

As shown in Figure 2C,D, the coculture system from the 24-well Transwell experiments showed that the presence of BEL-7402 cells in the lower chambers significantly caused both untreated and miR101-MSCs to permeate the membrane compared to the permeation in the absence of BEL-7402 cells in the lower chambers, thereby confirming the tropic nature of MSCs to HCCs. Furthermore, when the BEL-7402 cells were seeded in the lower chamber, there was a significantly decreased number of permeated cells in the miR101-MSCs group compared to the untreated MSCs group, which indicated that the tropism of MSCs to HCCs was slightly impaired by encapsulation of the LbL film.

\section{miR101-MSCs inhibited the proliferation and promoted apoptosis of BEL-7402 in vitro}

To evaluate the anti-tumor potential of miR101-LbLMSCs in vitro, the proliferation of BEL-7402 cells within the lower chambers of the coculture system was investigated by BrdU. As shown in Figure $3 A, B$, there was no significant difference in BEL-7402 cell proliferation between the Ctl-MSCs group and the untreated MSCs group, which indicated that the components (alginate and gelatin) used to encapsulate MSCs by LbL self-assembly did not affect the proliferation of BEL-7402 cells, while the miR101-MSCs significantly inhibited the proliferation of BEL-7402 cells, compared to both Ctl-MSCs and untreated MSCs, which can be attributed to the release of miR101. Further study showed that the expression of PCNA in BEL-7402 cells was significantly suppressed by the treatment of miR101-MSCs, compared to the treatment of Ctl-MSCs or untreated MSCs; while there was similar expression of PCNA in BEL-7402 cells between the Ctl-MSCs group and the untreated MSCs group (Figure 3C), which was consistent with the result of BrdU assay.

In addition to proliferation, the apoptosis of BEL-7402 cells in the lower Transwell chambers was also assayed using flow cytometry by annexin-V/PI double staining. 

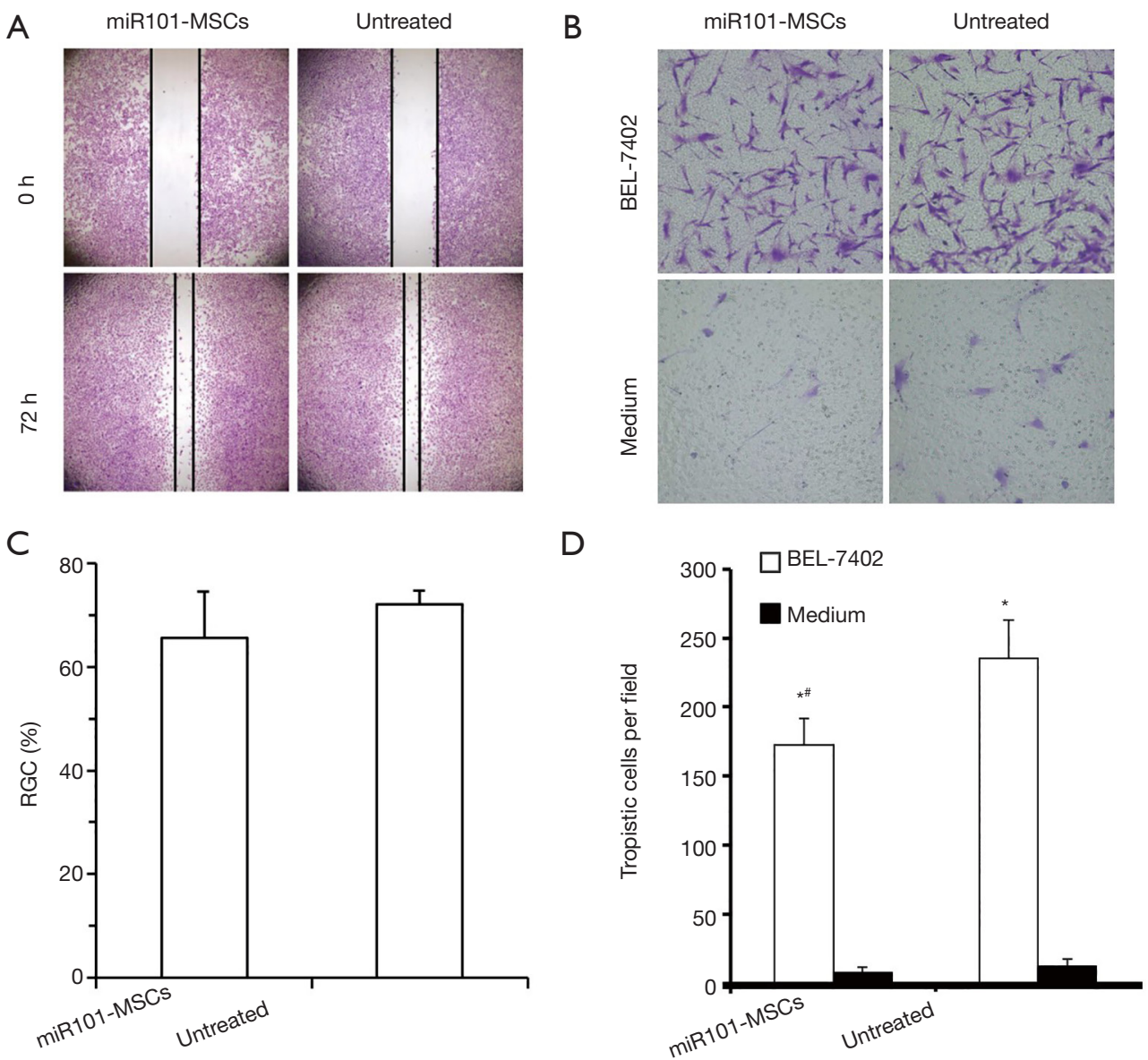

Figure 2 LbL film coating slightly impaired the tumor tropism of MSCs. (A) Representative images of scratch assays of MSCs collected at 0 $\mathrm{h}$ and $72 \mathrm{~h}$ to assess cell migration. (B) The rate of gap closure (RGC) in the scratch assay was calculated. The Transwell coculture system of MSCs and BEL-7402 cells was performed to detect the tropism of miR101-MSCs or untreated MSCs to BEL-7402 cells, and the cells that permeated to the lower surface of the upper chamber were stained (C) and quantified (D). *, P<0.05 compared with the same MSCs without BEL-7402 cells in the lower chamber; ${ }^{\prime}, \mathrm{P}<0.05$ compared with the untreated MSCs with BEL-7402 cells in the lower chamber.

The results showed that miR101-MSCs significantly induced BEL-7402 apoptosis compared to the Ctl-MSCs and the untreated MSCs (Figure 3D,E). Accordingly, immunoblotting showed that the expression of caspase-3 increased significantly in BEL-7402 cells treated by miR101-MSCs compared to those treated by Ctl-MSCs and untreated MSCs (Figure 3C).

\section{Discussion}

Therapeutic microRNA has a broad spectrum of anticancer activity, but it is also limited in its clinical applications. In this study, based on the tumor-tropism of MSCs and LbL self-assembly, we first developed a new microRNA delivery system. Our findings showed that gelatin and alginatemiR101 can be self-assembled on the surface of MSCs to form a thin film, and the miR101-loaded MSCs can grow in vitro in a spindle-like morphology. Furthermore, miR101-MSCs showed good tumor-tropism to hepatoma carcinoma cells and therapeutic effects on HCC, reflected by decreased proliferation and increased apoptosis of hepatoma carcinoma cells in the coculture system consisting of miR101-MSCs and BEL-7402 cells.

A previous study has reported that the LbL self-assembly technique can be used to fabricate a gelatin and alginate thin film, with a depth of $6 \pm 2.3 \mathrm{~nm}$, that can coat the surface of NSCs (25). In the current study, a similar depth of film encapsulated on the surface of MSCs, which can explain the 
A
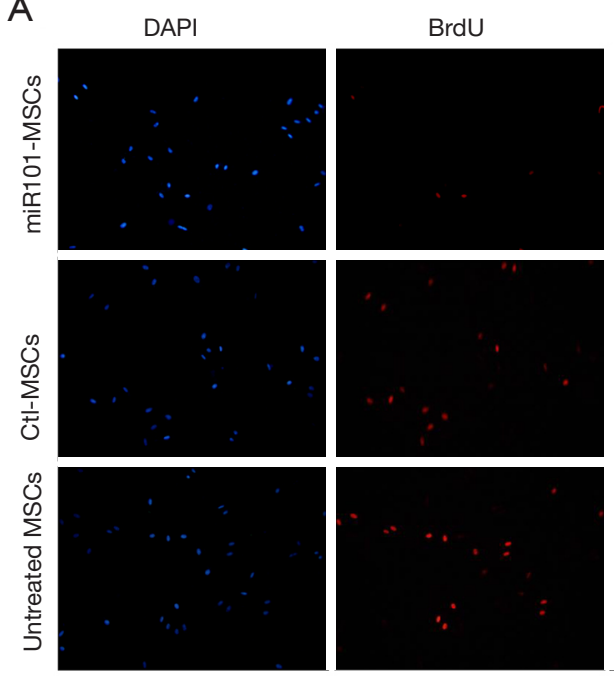

D
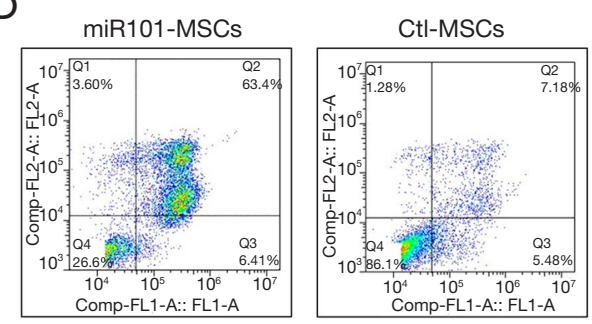

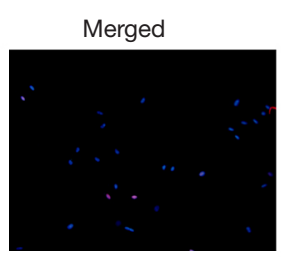

B
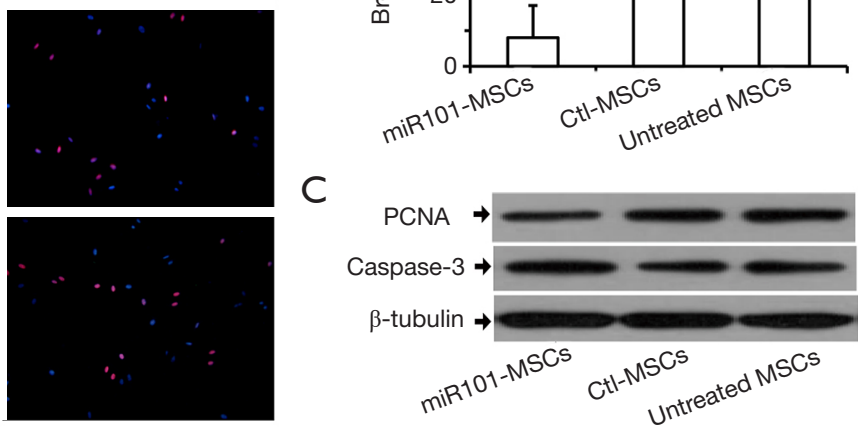

Untreated MSCs
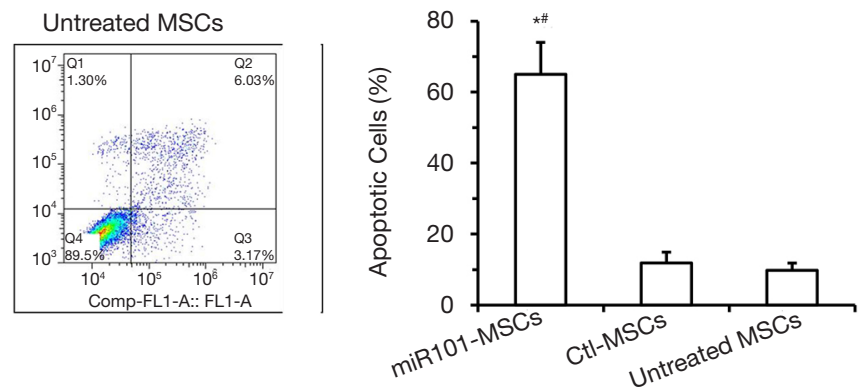

Figure 3 MiR101-MSCs inhibited proliferation and promoted apoptosis in BEL-7402 cells in vitro. MSCs and BEL-7402 cells were cocultured in a Transwell coculture system. The proliferation and apoptosis of BEL-7402 cells were detected by the BrdU incorporation assay and the annexin-V/PI apoptosis detection kit, respectively, and further confirmed by immunoblotting. Representative images of BrdU+ cells (A) and the quantified percentage of BrdU+ cells in 10 random fields (B) are shown. (C) The expression of PCNA and Caspase-3 in BEL-7402 cells were detected by immunoblotting. The cells stained by annexin-V/PI were analyzed by flow cytometry (D), followed by a statistical analysis (E). *, $\mathrm{P}<0.05$ vs. Ctl-MSCs; ${ }^{*}, \mathrm{P}<0.05$ vs. MSCs.

slower attachment of miR101-MSCs.

Zeta potential was commonly used to determine the formation of LbL films on cells or microparticles $(25,30,31)$. During the self-assembly process, the zeta potential altered alternatively based on the addition of each oppositely charged layer, which can demonstrate successful LbL assembly on the core. In the current study, a positive zeta potential value of approximately $5 \mathrm{mV}$ was obtained after the gelatin was assembled on the surface of MSCs, while the assembly of alginate-miR101 resulted in a negative zeta potential value of approximately $40 \mathrm{mV}$, which agrees well with the previous studies $(25,29)$.

The tumor-tropic capacity of MSCs mediated by the interaction between chemokines and $\mathrm{C}-\mathrm{X}-\mathrm{C}$ chemokine receptors (CXCR) was essential for the use of MSCs to deliver anticancer agents. Our results showed that the HCC tropism of the miR101-MSC group was well preserved, despite being poorer than that of the untreated MSCs, while the thin LbL film on the MSCs had no influence on the migratory capacity of MSCs. It is reasonable to believe that the tropism of LbL-MSCs was slightly weakened by the impaired chemokine/CXCR signaling resulting from the thin LbL film on the cell surface.

MiR101 has been reported to inhibit cell proliferation and induce cell apoptosis in many types of tumors, including breast, bladder, gastric and liver cancer (32-35). Our experimental results indicated that miR101 release from miR101-MSCs could result in the suppression of 
hepatoma carcinoma cell proliferation and induce the apoptosis of hepatoma carcinoma cells. A body of previous studies showed that treatment with nanoparticles coated with LbL films containing siRNA could effectively inhibit the expression of targeting genes in cancer cells, resulting in decreased cell viability and increased cell apoptosis (36-38). These previous studies agree with our findings that therapeutic RNA can be delivered to cancer cells by LbL technology using the proper core. However, it should be noted that the RNA encapsulated within the nanoparticle was taken into cells along with the nanoparticle (39), while the miR101 coated on the MSCs was endocytosed into tumor cells alone after release from MSCs.

In summary, miR101-MSCs provided a safer and more effective means of targeting miR101 delivery to HCC based on the tropism of MSCs and LbL self-assembly. Further studies are needed to optimize this novel delivery method and to investigate its therapeutic effect on HCC in vivo.

\section{Acknowledgments}

Funding: This work was supported by the $12^{\text {th }}$ Five-Year key project of the army of China (No. BWS11C056).

\section{Footnote}

Conflicts of Interest: All authors have completed the ICMJE uniform disclosure form (available at http://dx.doi. org/10.21037/tcr-19-1378b). The authors have no conflicts of interest to declare.

Ethical Statement: The authors are accountable for all aspects of the work in ensuring that questions related to the accuracy or integrity of any part of the work are appropriately investigated and resolved. The ethics approval is not required because no primary data collected from humans or animal.

Open Access Statement: This is an Open Access article distributed in accordance with the Creative Commons Attribution-NonCommercial-NoDerivs 4.0 International License (CC BY-NC-ND 4.0), which permits the noncommercial replication and distribution of the article with the strict proviso that no changes or edits are made and the original work is properly cited (including links to both the formal publication through the relevant DOI and the license). See: https://creativecommons.org/licenses/by-nc-nd/4.0/.

\section{References}

1. Shirjang S, Mansoori B, Asghari S, et al. MicroRNAs in cancer cell death pathways: Apoptosis and necroptosis. Free Radic Biol Med 2019;139:1-15.

2. Cao K, Li J, Zhao Y, et al. miR-101 Inhibiting Cell Proliferation, Migration and Invasion in Hepatocellular Carcinoma through Downregulating Girdin. Mol Cells 2016;39:96-102.

3. Lv X, Li J, Yang B. Clinical effects of miR-101 on prognosis of hepatocellular carcinoma and carcinogenic mechanism of anti-miR-101. Oncol Rep 2016;36:2184-92.

4. Xu F, Liao JZ, Xiang GY, et al. MiR-101 and doxorubicin codelivered by liposomes suppressing malignant properties of hepatocellular carcinoma. Cancer Med 2017;6:651-61.

5. Sun W, Zhang Q, Wu Z, et al. miR-101-3p sensitizes hepatocellular carcinoma cells to oxaliplatin by inhibiting Beclin-1-mediated autophagy. Int J Clin Exp Pathol 2019;12:2056-65.

6. Merhautova J, Demlova R, Slaby O. MicroRNA-Based Therapy in Animal Models of Selected Gastrointestinal Cancers. Front Pharmacol 2016;7:329.

7. Perepelyuk M, Thangavel C, Liu Y, et al. Biodistribution and Pharmacokinetics Study of siRNA-loaded AntiNTSR1-mAb-functionalized Novel Hybrid Nanoparticles in a Metastatic Orthotopic Murine Lung Cancer Model. Mol Ther Nucleic Acids 2016;5:e282.

8. Lv H, Zhang S, Wang B, et al. Toxicity of cationic lipids and cationic polymers in gene delivery. J Control Release 2006;114:100-9.

9. Beyerle A, Braun A, Merkel O, et al. Comparative in vivo study of poly(ethylene imine)/siRNA complexes for pulmonary delivery in mice. J Control Release 2011;151:51-6.

10. Chollet P, Favrot MC, Hurbin A, et al. Side-effects of a systemic injection of linear polyethylenimine-DNA complexes. J Gene Med 2002;4:84-91.

11. Xie Y, Murray-Stewart T, Wang Y, et al. Self-immolative nanoparticles for simultaneous delivery of microRNA and targeting of polyamine metabolism in combination cancer therapy. J Control Release 2017;246:110-9.

12. Tivnan A, Orr WS, Gubala V, et al. Inhibition of neuroblastoma tumor growth by targeted delivery of microRNA-34a using anti-disialoganglioside GD2 coated nanoparticles. PLoS One 2012;7:e38129.

13. Masters JC, Nickens DJ, Xuan D, et al. Clinical toxicity of antibody drug conjugates: a meta-analysis of payloads. Invest New Drugs 2018;36:121-35. 
14. Suryaprakash S, Lao YH, Cho HY, et al. Engineered Mesenchymal Stem Cell/Nanomedicine Spheroid as an Active Drug Delivery Platform for Combinational Glioblastoma Therapy. Nano Lett 2019;19:1701-5.

15. Xu M, Asghar S, Dai S, et al. Mesenchymal stem cells-curcumin loaded chitosan nanoparticles hybrid vectors for tumor-tropic therapy. Int J Biol Macromol 2019;134:1002-12.

16. Sasportas LS, Kasmieh R, Wakimoto H, et al. Assessment of therapeutic efficacy and fate of engineered human mesenchymal stem cells for cancer therapy. Proc Natl Acad Sci U S A 2009;106:4822-7.

17. Porada CD, Almeida-Porada G. Mesenchymal stem cells as therapeutics and vehicles for gene and drug delivery. Adv Drug Deliv Rev 2010;62:1156-66.

18. Mego M, Cholujova D, Minarik G, et al. CXCR4-SDF-1 interaction potentially mediates trafficking of circulating tumor cells in primary breast cancer. BMC Cancer 2016;16:127.

19. Zhang Q, Xiang W, Yi DY, et al. Current status and potential challenges of mesenchymal stem cell-based therapy for malignant gliomas. Stem Cell Res Ther 2018;9:228.

20. Kucerova L, Matuskova M, Pastorakova A, et al. Cytosine deaminase expressing human mesenchymal stem cells mediated tumour regression in melanoma bearing mice. $\mathrm{J}$ Gene Med 2008;10:1071-82.

21. Kucerova L, Altanerova V, Matuskova M, et al. Adipose tissue-derived human mesenchymal stem cells mediated prodrug cancer gene therapy. Cancer Res 2007;67:6304-13.

22. Yoshida K, Hashide R, Ishii T, et al. Layer-by-layer films composed of poly(allylamine) and insulin for $\mathrm{pH}$ triggered release of insulin. Colloids Surf B Biointerfaces 2012;91:274-9.

23. Shen HJ, Shi H, Xie M, et al. Biodegradable chitosan/ alginate BSA-gel-capsules for $\mathrm{pH}$-controlled loading and release of doxorubicin and treatment of pulmonary melanoma. J Mater Chem B 2013;1:3906-17.

24. Crouzier T, Ren K, Nicolas C, et al. Layer-By-Layer Films as a Biomimetic Reservoir for rhBMP-2 Delivery: Controlled Differentiation of Myoblasts to Osteoblasts. Small 2009;5:598-608.

25. Li W, Guan T, Zhang X, et al. The Effect of Layerby-Layer Assembly Coating on the Proliferation and Differentiation of Neural Stem Cells. ACS Appl Mater Interfaces 2015;7:3018-29.
26. Shutava TG, Balkundi SS, Vangala P, et al. Layer-by-LayerCoated Gelatin Nanoparticles as a Vehicle for Delivery of Natural Polyphenols. Acs Nano 2009;3:1877-85.

27. Carter JL, Drachuk I, Harbaugh S, et al. Truly nonionic polymer shells for the encapsulation of living cells. Macromol Biosci 2011;11:1244-53.

28. Han N, Zhang F, Li G, et al. Local application of IGFBP5 protein enhanced periodontal tissue regeneration via increasing the migration, cell proliferation and osteo/ dentinogenic differentiation of mesenchymal stem cells in an inflammatory niche. Stem Cell Res Ther 2017;8:210.

29. Liu G, Li L, Huo D, et al. A VEGF delivery system targeting MI improves angiogenesis and cardiac function based on the tropism of MSCs and layer-by-layer selfassembly. Biomaterials 2017;127:117-31.

30. Kakade S, Manickam DS, Handa H, et al. Transfection activity of layer-by-layer plasmid DNA/poly(ethylenimine) films deposited on PLGA microparticles. Int J Pharm 2009;365:44-52.

31. Hwang J, Choi D, Choi M, et al. Synthesis and Characterization of Functional Nanofilm-Coated Live Immune Cells. ACS Appl Mater Interfaces 2018;10:17685-92.

32. Guan H, Dai Z, Ma Y, et al. MicroRNA-101 inhibits cell proliferation and induces apoptosis by targeting EYA1 in breast cancer. Int J Mol Med 2016;37:1643-51.

33. Chen L, Long Y, Han Z, et al. MicroRNA-101 inhibits cell migration and invasion in bladder cancer via targeting FZD4. Exp Ther Med 2019;17:1476-85.

34. Wu F, Huang W, Yang L, et al. MicroRNA-101-3p regulates gastric cancer cell proliferation, invasion and apoptosis by targeting PIM 1 expression. Cell Mol Biol (Noisy-le-grand) 2019;65:118-22.

35. Liu Y, Tan J, Ou S, et al. MicroRNA-101-3p suppresses proliferation and migration in hepatocellular carcinoma by targeting the HGF/c-Met pathway. Invest New Drugs 2020;38:60-9.

36. Labala S, Jose A, Chawla SR, et al. Effective melanoma cancer suppression by iontophoretic co-delivery of STAT3 siRNA and imatinib using gold nanoparticles. Int J Pharm 2017;525:407-17.

37. Choi KY, Correa S, Min J, et al. Binary Targeting of siRNA to Hematologic Cancer Cells In Vivo using Layer-by-Layer Nanoparticles. Adv Funct Mater 2019;29:1900018.

38. Wang X, Liu K, Yang G, et al. Near-infrared light triggered photodynamic therapy in combination with gene 
therapy using upconversion nanoparticles for effective cancer cell killing. Nanoscale 2014;6:9198-205.

39. Shen J, Kim HC, Mu C, et al. Multifunctional gold

Cite this article as: $\mathrm{Li} \mathrm{C}, \mathrm{Li} \mathrm{X}, \mathrm{Li} \mathrm{F}$, Wang D, Wang J, Yan T, Sun B, Ren H. A microRNA delivery carrier for hepatic carcinoma therapy using layer-by-layer self-assembled mesenchymal stem cells. Transl Cancer Res 2020;9(9):5380-5389. doi: $10.21037 /$ tcr-19-1378b nanorods for siRNA gene silencing and photothermal therapy. Adv Healthc Mater 2014;3:1629-37. 